\title{
Aspergillus fumigatus fungus ball in the native lung after single lung transplantation
}

\author{
Bola fúngica por Aspergillus fumigatus no pulmão nativo \\ após transplante unilateral de pulmão \\ Fernando Ferreira Gazzoni, Bruno Hochhegger, \\ Luiz Carlos Severo, José Jesus Camargo
}

\section{To the Editor:}

A 49-year-old woman underwent right lung transplantation due to pulmonary emphysema, with favorable evolution in the early postoperative period. A year later she was readmitted to our department with productive cough. During that admission, the patient was treated for cytomegalovirus pneumonia and received broadspectrum antibacterial therapy.

At outpatient follow-up, cavities appeared in the native lung, which gradually increased in size. Ten months later, she was admitted for the resection of a hyperinflated cavity. Chest $\mathrm{X}$-rays showed an increase in the cavity in the left upper lobe with herniation of the lung and compression of the transplanted lung. Chest HRCT at various positions showed a round mass with soft tissue density within a lung cavity that moved when the patient changed position, thus strengthening the hypothesis of a fungus ball (Figure 1). Bullectomy was performed, and the histopathologic examination showed fungal colonization by Aspergillus fumigatus in emphysematous bullae and bronchiectasis. She was treated with itraconazole and had a satisfactory response.

Lung transplantation has become an acceptable treatment option for many end-stage lung diseases and could be single or double. ${ }^{(1,2)}$ However, Aspergillus sp. infections continue to be an important cause of morbidity and mortality in these patients. Aspergillus sp. is an ubiquitous fungus that can cause clinical entities of varying severity, such as asymptomatic colonization, aspergilloma, tracheobronchitis, active parenchymal disease, and angioinvasive aspergillosis. ${ }^{(1-6)}$

Airway colonization is a common occurrence in such patients because of the exposure of the transplanted lung to the environment and impaired local host defenses, including mucociliary clearance. In addition, colonization of the native lung, which commonly occurs in end-stage lung disease, is an important source of post-transplantation aspergillosis in single lung transplantation recipients. Aspergillus sp. colonization has also been related to cytomegalovirus infection and chronic rejection. ${ }^{(1-4)}$

Patients who undergo unilateral transplantation are often older and have a higher prevalence of COPD as an underlying disease, a condition that might predispose to airway colonization by Aspergillus sp. ${ }^{(1-3)}$

The most accurate way to perform the diagnosis is the demonstration of characteristic, acute branching, broad, septate hyphae showing zones of growth in biopsy/surgical/autopsy specimens and positive cultures for Aspergillus sp..$^{(3,5,6)}$

Our patient presented with Aspergillus fumigatus fungal ball (aspergilloma) in emphysematous bullae and bronchiectasis in the native lung 26 months after transplantation, with a satisfactory response to medical and surgical treatment. She also had a history of cytomegalovirus infection one year after transplantation as another risk factor. The diagnosis was made through imaging and evaluation of surgical specimens. In fact, aspergilloma affecting the native lung in single lung transplantation recipients has been reported only rarely in retrospective studies. . $^{(3,4)}$

Aspergilloma is characterized by Aspergillus sp. infection without tissue invasion. It leads to the conglomeration of intertwined fungal hyphae mixed with mucus and cellular debris within a pre-existing pulmonary cavity, bulla, or ectatic bronchus. The most common underlying causes are tuberculosis and sarcoidosis. Although patients might remain asymptomatic, the most common clinical manifestation is hemoptysis. ${ }^{5,6)}$ Risk factors for a poor prognosis of aspergilloma include the severity of the underlying lung disease, increase in size or in the number of lesions on 


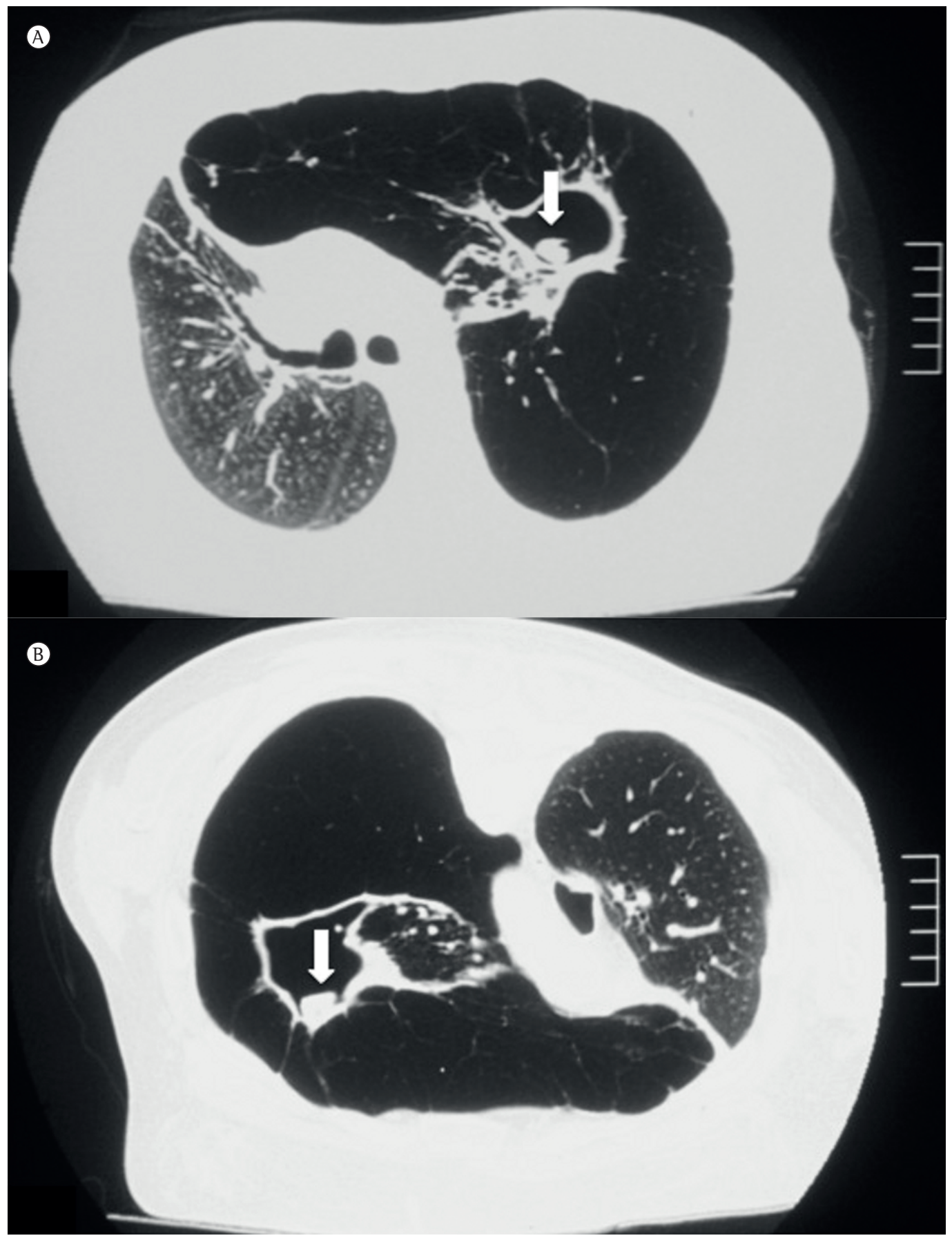

Figure 1 - Axial HRCT scans. In A, cavitary lung lesion in the left upper lobe filled with an opacity resembling a fungus ball (arrow). In B, a scan after moving the patient from the supine position to the prone position, demonstrating the motility of the mass (arrow).

chest X-rays, immunosuppression (including transplantation), increasing Aspergillus-specific lgG titles, recurrent large volume hemoptysis, and sarcoidosis. This highlights the importance of radiological findings, cultures, reviews of the level of immunosuppression, and environmental factors for the early diagnosis and prevention of further complications, such as angioinvasive disease. Treatment should be considered only when patients become symptomatic, usually with 
hemoptysis. There is no consensus on the best treatment approach; however, surgical resection of the cavity and removal of the fungus ball are usually indicated in patients with recurrent hemoptysis if their pulmonary function is sufficient to allow surgery. ${ }^{(4-6)}$

On CT scans and $\mathrm{X}$-rays, aspergillomas are characterized by the presence of a round or oval mass with soft tissue density within a lung cavity. The mass can be separated from the wall of the cavity by an air space of variable size, resulting in the "air crescent" sign. The aspergilloma usually moves when the patient changes position, as was seen in our case. Another finding of aspergillomas is the thickening of the cavity wall and adjacent pleura, which might be the earliest radiological sign..$^{(5,6)}$

In summary, the susceptibility of the native lung to Aspergillus sp. infections might be an additional factor to be considered in choosing the ideal transplantation procedure. In cases of single lung transplantation, radiological and clinical attention is especially directed to the transplanted organ. However, the native lung, structurally damaged, can be a nidus for Aspergillus sp. and provide a source of infection.

\section{Fernando Ferreira Gazzoni}

Radiologist, Department of Radiology, Porto Alegre Hospital de Clínicas, Porto Alegre, Brazil

\section{Bruno Hochhegger}

Thoracic Radiologist, Pulmonology Department, Santa Casa Sisters of

Mercy Hospital Complex, and Professor of Radiology, Federal University of
Health Sciences of Porto Alegre, Porto Alegre, Brazil

Luiz Carlos Severo

Associate Professor, Department of

Internal Medicine, Federal University of

Rio Grande do Sul, Porto Alegre, Brazil

José Jesus Camargo

Thoracic Surgeon, Department of

Pulmonology and Thoracic Surgery,

Santa Casa Sisters of Mercy Hospital

Complex, Porto Alegre, Brazil

\section{References}

1. Singh N, Husain S. Aspergillus infections after lung transplantation: clinical differences in type of transplant and implications for management. J Heart Lung Transplant. 2003;22(3):258-66. http://dx.doi.org/10.1016/ S1053-2498(02)00477-1

2. McAdams HP, Erasmus JJ, Palmer SM. Complications (excluding hyperinflation) involving the native lung after single-lung transplantation: incidence, radiologic features, and clinical importance. Radiology. 2001;218(1):23341. PMid: 11152808

3. Westney GE, Kesten S, De Hoyos A, Chapparro C, Winton T, Maurer JR. Aspergillus infection in single and double lung transplant recipients. Transplantation. 1996;61(6):915-9. http://dx.doi.org/10.1097/00007890-199603270-00013 PMid:8623160

4. Fitton TP, Bethea BT, Borja MC, Yuh DD, Yang SC, Orens JB, et al. Pulmonary resection following lung transplantation. Ann Thorac Surg. 2003;76(5):1680-5; discussion 1685-6.

5. Franquet T, Müller NL, Giménez A, Guembe P, de La Torre J, Bagué S. Spectrum of pulmonary aspergillosis: histologic, clinical, and radiologic findings. Radiographics. 2001;21(4):825-37. PMid:11452056

6. Kousha M, Tadi R, Soubani A0. Pulmonary aspergillosis: a clinical review. Eur Respir Rev. 2011;20(121):15674. http://dx.doi.org/10.1183/09059180.00001011 PMid:21881144 